\title{
The Disk of Our Galaxy
}

\author{
Delphine Russeil \\ LAM, Université de Provence, \\ F-13388, Marseille, France \\ email: delphine.russeil@oamp.fr
}

\begin{abstract}
We review our knowledge about the spiral structure of the disk of our Galaxy using tracers of star formation. These tracers reveal a 4 -arm picture of the Galaxy.
\end{abstract}

Keywords. Galaxy: structure, disk

Multi-wavelength views ( $\mathrm{UV}, \mathrm{CO}, \mathrm{H} \alpha$ ) of spiral galaxies as M31, M51 and M83 show that the arms are well traced by young objects (OB stars, giant molecular clouds, HII regions). The mid-infrared Spitzer-24 $\mu \mathrm{m}$ image of M31 shows well traced arms, but in the near-infrared the arm contrast is low. For an observer inside the Galaxy, locating the arms can be confused by the numerous small filaments seen in Spitzer- $8 \mu \mathrm{m}$ images of M31 and M83. The HI emission, well known to extend far away the optical disk, is used to probe the external part of the disk. A new aspect comes from the recent 70, 100 and 160 $\mu \mathrm{m}$ Herschel images of M51 in the far-infrared (emission coming from active star-forming complexes) which show that spiral arms are well traced at these wavelengths.

To study the structure of our Galaxy we use the same tracers. Our approach is to use star-forming complexes (composed of the parental molecular cloud associated with the OB stars and their HII regions) as tracer of the spiral arms (Russeil (2003)). This method enables us to reduce the spatial and kinematic spread of objects belonging to the same complex. The multi-wavelength information (HII region recombination lines, parental cloud molecular lines and OB stars spectro-photometric distance) allows a better determination of the systemic velocity and distance. We then confirmed the 4-arm model and showed that the Carina-Sagittarius arm is a major optical arm. An updated view of the 4-arm structure is done by Hou et al. (2009) who also show a good connection with the HI arms seen in the outer part by Levine et al. (2006). From NIR star counts and FIR emission profiles, giving the arm tangencies, Benjamin (2008) and Drimmel (2000) find that the Scutum-Crux and the Perseus arms are the 2 major arms. But plotting the tangent directions from Hou et al. (2009) and Russeil (2003) one can see that these profiles can be compatible with the 4 -arm models.

The arm locations should be improved thanks to new distance determination (GAIA stellar parallaxes, maser parallaxes and infrared spectro-photometric methods) and to recent/incoming multi-wavelength surveys which allow a systematic resolution of the distance ambiguity problem and a better definition of the star-forming complexes.

\section{References}

Benjamin, R. 2008, Massive star Formation, ASP Conf. Series, 387, 735

Drimmel, R. 2000, A\&SA, 358, L13

Hou, L., Han, J., \& Shi, W. 2009, A\&A, 499, 473

Levine, E., Blitz, L., \& Heiles, C. 2006, Science, 312, 1773

Russeil, D. 2003, A\& $A, 397,133$ 